\title{
The Italian Research Group for Gastric Cancer (GIRCG) guidelines for gastric cancer staging and treatment: 2015
}

\author{
Giovanni De Manzoni ${ }^{1}$ - Daniele Marrelli ${ }^{1}$ Gian Luca Baiocchi ${ }^{1}$. \\ Paolo Morgagni $^{1} \cdot$ Luca Saragoni $^{1} \cdot$ Maurizio Degiuli $^{1} \cdot$ Annibale Donini $^{1}$ • \\ Uberto Fumagalli $^{1}$ - Maria Antonietta Mazzei ${ }^{1}$ - Fabio Pacelli ${ }^{1}$ Anna Tomezzoli ${ }^{1}$. \\ Mattia Berselli $^{1} \cdot$ Filippo Catalano $^{1}$. Alberto Di Leo ${ }^{1} \cdot$ Massimo Framarini $^{1}$ • \\ Simone Giacopuzzi ${ }^{1}$ - Luigina Graziosi ${ }^{1}$ - Alberto Marchet $^{1} \cdot$ Mario Marini ${ }^{1}$. \\ Carlo Milandri $^{1}$ - Gianni Mura ${ }^{1}$ - Elena Orsenigo ${ }^{1}$ - Vittorio Quagliuolo ${ }^{1}$. \\ Stefano Rausei ${ }^{1} \cdot$ Riccardo Ricci $^{1} \cdot$ Fausto Rosa $^{1}$ - Giandomenico Roviello ${ }^{1}$. \\ Andrea Sansonetti ${ }^{1} \cdot$ Giovanni Sgroi $^{1} \cdot$ Guido Alberto Massimo Tiberio $^{1}$. \\ Giuseppe Verlato ${ }^{1} \cdot$ Carla Vindigni $^{1} \cdot$ Riccardo Rosati $^{1} \cdot$ Franco Roviello $^{1}$
}

Received: 18 February 2016/Accepted: 1 May 2016/Published online: 2 June 2016

(C) The International Gastric Cancer Association and The Japanese Gastric Cancer Association 2016

\begin{abstract}
This article reports the guidelines for gastric cancer staging and treatment developed by the GIRCG, and contains comprehensive indications for clinical management, including radiological, endoscopic, surgical, pathological, and oncological paths.
\end{abstract}

Keywords Gastric cancer · Guidelines · Staging · Surgery · Chemotherapy · Lymphadenectomy · Palliation · Eesophagus-gastric junction tumors · GIST

\section{Introduction}

Gastric cancer (GC), despite its declining incidence, is still the third cancer-related cause of death after lung and liver neoplasms [1]. Although surgery remains the mainstay of therapy, in recent years there has been relevant progress in endoscopic treatment of early forms and in neoadjuvant, adjuvant, and palliative chemotherapy of advanced cancers. Furthermore, radiological and pathological protocols have been standardized. Thus, a multidisciplinary team is required for the correct management of patients, from preoperative staging to follow-up.

On behalf of GIRCG.

Daniele Marrelli

daniele.marrelli@unisi.it

1 GIRCG Secretary: Department of Medicine, Surgery and Neurosciences, University of Siena, Policlinico Le Scotte, Viale Bracci, 53100 Siena, Italy
From an historical perspective, Italian surgeons were among the first in the West to acknowledge the indications of Eastern centers; because of the high incidence of this tumor in their countries, the Japanese surgeons developed a surgical approach based on extended (D2) and super-extended (D3) lymphadenectomy, whereas the intervention more frequently performed in Europe and in the United States (US) provided for a limited lymphadenectomy (D1). This disparity gave rise to a scientific conflict that rested on an impressive difference in long-term survival (overall, 5 -year survival rate of a patient with GC was about $75 \%$ in Japan [2] and $25 \%$ in Europe [3]). In this context, a number of Italian surgeons in the 1980s started concentrating their efforts on more meticulous and aggressive nodal clearance and on providing a contribution to the worldwide dissemination of Japanese therapy and its results.

The Italian Research Group for Gastric Cancer (GIRCG) is a multidisciplinary research group, officially founded in 2001, that includes clinicians with recognized expertise in GC diagnosis, care, and research from more than 25 specialized centers in Italy. The aim of GIRCG is to obtain results similar to those reported by Eastern centers in terms of recurrence rate and survival. GIRCG involves a variety of medical professionals, from surgeons, pathologists, gastroenterologists, medical oncologists, and radiologists to nutritionists and statisticians, who all practice within the modern concepts of a multidisciplinary approach. The main targets of the group are the standardization of surgical treatment and extended lymphadenectomy, pathological assessment, clinical staging, and multimodal treatment of 
GC in Italy, in surgical, endoscopic, pathological, and radiological training, as well as the conduction of clinical studies and translational research. A mean of three meetings per year are conducted to ensure a continuity of collaboration. In the past 10 years the GIRCG published 45 papers in indexed journals and an international book [4], organized 12 workshops and one post-university master class, and finally the 10th International Gastric Cancer Congress in June 2013 in Verona. Several research studies are still ongoing.

In September 2013, surgical guidelines for GC were issued by the GIRCG and the Italian Society of Surgery (SIC), and at the end of 3 months, the Web-based and Delphi method-based Consensus Conference [5]. The final version included nine statements (Staging, Endoscopic Treatment, Neoadjuvant Therapy, Extent of Gastric Resection, Lymphadenectomy, Associated Resections, Palliative Therapy, Mini-invasive Surgery, Follow-up), which were approved in plenary session during the 105th SIC National Congress, October 2013, in Turin. Starting from these statements, in the following months a Commission was established inside the GIRCG, with the aim to translate those results into comprehensive indications for clinical management, including radiological, endoscopic, surgical, pathological, and oncological paths. The result is herein disclosed under the title of "GIRCG guidelines for GC staging and treatment: 2015", and should be reevaluated in 3 years. The present guidelines have not already been published elsewhere, even in the Italian language or other forms. The present paper has been approved by the Scientific Committee of the GIRCG.

\section{Diagnosis and staging}

Diagnosis of GC is usually done - and should in every case be confirmed-by upper gastrointestinal (GI) endoscopy. Basic information to be provided by endoscopy are location [upper, middle, lower third, esophagogastric junction (EGJ), divided in Siewert type I, II, or III], size, macroscopic appearance, and actual complications (obstruction/bleeding). Biopsies from the tumor should always be taken to confirm histology and to classify into potentially useful classifications [Lauren histotype; World Health Organization (WHO); see later]. Chromoendoscopy and biopsies of the gastric mucosa far from the tumor may be useful to exclude multifocal disease. The suspicion of Barrett's esophagus should be specified and eventually confirmed by separate biopsies.

The pretreatment staging of GC should include in all cases a contrast-enhanced thoracoabdominal multidetectorrow computed tomography (MDCT) with 16 or more rows. The MDCT examination should be performed with a spiral technique, using a dedicated protocol optimized to detect serosal invasion and minimal peritoneal disease, and images should be analyzed by an experienced reader (see Appendix). Endoscopic ultrasound (EUS) may improve the diagnostic accuracy of T-stage, particularly in discriminating $\mathrm{T} 1 \mathrm{a}$ from $\mathrm{T} 1 \mathrm{~b}$ or $\mathrm{T} 2$, or in case of an inadequate $\mathrm{CT}$ examination; however, it is not strictly necessary in advanced forms, whereas it is formally indicated in the selection of patients for endoscopic treatment. Staging laparoscopy is also not strictly required, but it is recommended in cases deemed to be at risk of peritoneal carcinomatosis not visible or doubtful at CT examination. Staging laparoscopy is required also in many randomized clinical trials of adjuvant and neoadjuvant therapy. The cytological examination of peritoneal lavage, although limited by a low sensitivity, is a useful completion of the final pathological staging.

\section{Endoscopic treatment of early gastric cancer (EGC)}

As early gastric cancer (EGC) has an excellent prognosis, endoscopic procedures have been increasingly adopted for the treatment of selected cases with low risk for nodal metastases, with the aim of avoiding greater than necessary morbidity and mortality related to gastrectomy. The GIRCG recognizes the criteria for appropriate endoscopic therapy of EGC reported in the gastric cancer treatment guidelines 2010, published by the Japanese Gastric Cancer Association (JGCA) [6]: the absolute criteria for standard treatment [including both endoscopic mucosal resection (EMR) and endoscopic submucosal dissection (ESD] are differentiatedtype adenocarcinoma, no ulcerative findings (UL(-)), depth of invasion clinically diagnosed as T1a (mucosal stage), and diameter not greater than $2 \mathrm{~cm}$; the expanded criteria, to be proposed as an investigational treatment (only ESD should be employed), are tumors clinically diagnosed as T1a and (a) of differentiated type, UL(-), but greater than $2 \mathrm{~cm}$ in diameter, or (b) of differentiated type, $\mathrm{UL}(+)$, and not greater than $2 \mathrm{~cm}$ in diameter. The resection is judged as curative when all the following conditions are fulfilled: en bloc resection, tumor size not greater than $2 \mathrm{~cm}$, histology of intestinal differentiated type, pT1a, negative horizontal (lateral) margin (HM0), negative vertical margin (VM0), and no lymphovascular invasion [7]. It is reasonable to treat EGC that meet the aforementioned characteristics by endoscopic techniques (EMR or ESD) only in experienced, high-volume centers. Extended criteria may be proposed only to patients who accept to undergo long-term endoscopic surveillance or to participate in investigational programs. In centers with low volume of endoscopic advanced procedures, gastrectomy remains the gold standard for treatment of EGC [8]. 


\section{Neoadjuvant treatment}

The indication to perioperative chemotherapy should be considered and discussed within a multidisciplinary team in every case of locally advanced GC. The randomized studies MAGIC [9] and FNCLCC [10] are the principal reference, in Europe, for integrated protocols: these studies have demonstrated a survival benefit for neoadjuvant and perioperative treatment in GC staged $>\mathrm{T} 1$ and/or $\mathrm{N}+$. In the MAGIC trial, the 5-year overall survival (OS) rates were $36 \%$ among those who received perioperative chemotherapy and $23 \%$ in the surgery group [hazard ratio (HR) $0.75, P=0.009$ ]. In the FNCLCC trial, the 5-year overall survival (OS) rate was $38 \%$ for patients in the perioperative chemotherapy group and $24 \%$ in the surgery-only group (HR $0.69, P=0.02$ ). The corresponding 5-year disease-free survival (DFS) rates were $34 \%$ and $19 \%$ (HR $0.65, P=0.003$ ), respectively. Multidisciplinary evaluation must consider several aspects that are important in the choice of an individualized treatment plan: an accurate preoperative stage of GC is difficult to achieve; the symptoms related to advanced tumors, obstruction or bleeding, may contraindicate neoadjuvant treatment; very limited data exist on the possibility to predict the response of a single neoplasm to neoadjuvant treatment; only response to treatment determines the survival advantage. Serosal infiltrating tumors, cancers with bulky (enlarged, clearly metastatic) nodes, or Bormann type 4 cancers are a common indication for neoadjuvant treatment, mainly with the aim of increasing the $\mathrm{R} 0$ resectability rate [11-13]. At present, there are doubts about the response rate of signet-ring cell tumors to neoadjuvant treatment, because of a presumed intrinsic chemoresistance of these cancers. It is possible that these cancers necessitate a different integrated treatment pathway. The GIRCG suggests considering a neoadjuvant treatment for GC $T \geq 3$ and/or with metastatic nodes on preoperative workup, because the 5 -year survival probability of $\mathrm{T} 1 / \mathrm{T} 2$ node-negative cases largely surpasses $80 \%$ in GIRCG series [14]. Selection of neoadjuvant treatments should take into consideration some elements that may determine collateral effects and related postoperative morbidity: patient age, for example, can be a parameter to decide the use of intensive regimens. There still remains to be defined the rate of postoperative morbidity directly related to neoadjuvant treatment and the most effective treatment between preoperative and perioperative schema.

\section{Surgical therapy}

\section{Resection}

Curative surgery is distinguished as standard gastrectomy (total or subtotal gastric resection and D2 lymphadenectomy), modified gastrectomy (the extent of gastric resection and/or lymphadenectomy is reduced compared to standard surgery), and extended gastrectomy (gastric resection plus surgical removal of adjacent involved organs and/or D2 plus lymphadenectomy).

A sufficient resection margin should be ensured when determining the resection line in gastrectomy with curative intent. A proximal margin of at least $3 \mathrm{~cm}$ is recommended for $\mathrm{T} 2$ or deeper tumors with an expansive growth pattern, and $5 \mathrm{~cm}$ is recommended for those with infiltrative growth pattern and diffuse Lauren histotype. When these rules cannot be respected, it is advisable to examine the proximal resection margin by frozen section. For tumors invading the esophagus, a $5-\mathrm{cm}$ margin is not necessarily required, but frozen section examination of the resection line is desirable to ensure an R0 resection. For T1 tumors, a gross resection margin of $2 \mathrm{~cm}$ should be obtained. When the tumor border is unclear, preoperative endoscopic marking of the tumor border by clips will be helpful for decision making regarding the resection line. Distal gastrectomy should be preferred when an adequate proximal resection margin can be obtained for distal tumors. Pancreatic or spleen invasion by tumor requiring pancreaticosplenectomy necessitates total gastrectomy regardless of tumor location. Total gastrectomy should be considered for tumors that are located along the greater curvature of the corpus (when there is not an adequate surgical margin) or the fundus.

After distal gastrectomy, Roux-en-Y reconstruction seems superior to Billroth I and Billroth II reconstructions in terms of functional outcomes and long-term endoscopic results; however, no clear conclusions are available in the literature, and the choice of the procedure could be based on the surgeon's experience $[15,16]$. After total gastrectomy, Roux-en-Y reconstruction remains the easiest solution, with satisfactory functional results.

Splenectomy is generally associated with an increased risk of postoperative complications in GC surgery. Final survival analysis of a randomized controlled trial (JCOG0110), designed to evaluate the role of splenectomy in total gastrectomy for proximal GC that does not invade the greater curvature, demonstrated significant noninferiority of spleen preservation [17]. Total gastrectomy with splenectomy should be recommended for tumors that are located along the greater curvature or when a macroscopic involvement of stations 4 sa or 10 is present.

Combined cholecystectomy for asymptomatic gallstone in GC surgery may be considered in young patients; otherwise, it is not clear if cholecystectomy is indicated in patients without gallstones; a recent GIRCG multicenter study showed no difference in medium-term outcome between patients receiving or not receiving prophylactic cholecystectomy [18]. 
The role of total omentectomy is still questionable, particularly for serosa-negative advanced GC. Removal of the greater omentum is usually integrated in the standard gastrectomy for $\mathrm{T} 3$ or deeper tumors. For T1/T2 tumors, the omentum more than $3 \mathrm{~cm}$ away from the gastroepiploic arcade may be preserved.

When the posterior gastric wall serosa is infiltrated by the tumor, removal of the inner peritoneal surface of the bursa omentalis may be performed to remove microscopic tumor deposits in the lesser sac. In T1/T2 tumors, bursectomy should be avoided to prevent injury to the pancreas and adjacent vessels. A small-scale randomized controlled trial (RCT) showed a trend toward improved survival after bursectomy for tumors in the middle or lower third and for pathologically serosa-positive tumors [19].

\section{Lymphadenectomy}

The GIRCG takes strictly into account the Guidelines of the JGCA for indications, surgical procedure, and classification of lymphadenectomy [6]. In particular, the following points are emphasized: the standard treatment for potentially curative resection is the D2, even after neoadjuvant treatment. Only in carefully selected cases (high-risk patients, early tumors not treatable by endoscopic resections) should more limited procedures be considered (D1, D1 plus). Otherwise, it is strictly necessary to follow the correct procedure of lymphadenectomy, with special reference, along with other perigastric nodes, to an accurate and complete removal of infrapyloric (station 6), right paracardial (station 1), left gastric artery (station 7), celiac axis (station 9), hepatic artery (station 8a), splenic artery (station $11 \mathrm{p} / \mathrm{d}$ ), and hepatoduodenal ligament (12a) nodes. It is also emphasized that in Italy the preoperative diagnosis of early forms is often unreliable, and the incidence of Lauren diffuse histotype, which is associated with a higher risk of lymph node metastases even in early forms, is high [20]. The D2 plus, which involves the lymphadenectomy of posterior stations $(8 \mathrm{p}, 12 \mathrm{p} / \mathrm{b}, 13)$, station $14 \mathrm{v}$, and the additional removal of paraaortic nodes $(16 \mathrm{a} 2,16 \mathrm{~b} 1)$, may be justified in patients at high risk of metastases at these stations (advanced tumors of the upper third, advanced tumors and diffuse histotype located in the distal two-thirds of the stomach). However, these procedures should be performed in centers specialized with the D2, or in clinical trials [21]. Lymph node mapping on the fresh specimen is advisable to check the quality control of lymphadenectomy and potentially increase the number of examined nodes, thus allowing a more correct staging of the disease.

\section{Minimally invasive resective surgery (MIS)}

Laparoscopic gastric resection for GC is an option that should be considered in patients with EGC: this approach carries advantages in terms of reduction of postoperative stay, postoperative pain, and return to normal activities. However, the results of MIS in terms of quality of life and long term survival are still under evaluation [22, 23]. Preliminary data seem to indicate that laparoscopic surgery is feasible also for AGC, but solid data on the advantages and oncological efficacy of this approach coming from randomized trials are lacking, and the presence of a serosal cancer should still be considered a contraindication to MIS. There are some limitations to a diffuse application of these data, which come mainly from eastern RCT, including patients with BMI generally lower than those of Western patients, with fewer comorbidities and with tumors with a different biological behavior [24]. There would also be a problem concerning the learning curve for this procedure, which requires a caseload difficult to be reached in a short time in regions with a low prevalence of GC; in most of the studies coming from the East, a "laparoscopically assisted" technique was used, and such results are not directly transferrable to a totally laparoscopic approach. Finally, beyond disease stage it should be considered that the available evidence concerns only subtotal resections: total gastrectomy includes some technical steps that are not standardized and which still make the procedure uncommon.

\section{Pathological report}

EGC is a malignant epithelial neoplasia limited to the mucosa and/or submucosa [25]. From a macroscopic point of view, EGCs are divided into three main types according to their endoscopic appearance: type 1 (protruding), type 2 (superficial), type 3 (excavated). Kodama's classification should also be mentioned, as it could provide additional prognostic implications [26]. Advanced carcinomas should be classified into four macroscopic types according to the criteria proposed by Borrmann: polypoid, fungating, ulcerated, and infiltrative. The diffuse variant may affect most of the stomach and is commonly called linitis plastica or leather bottle stomach. The most widely used histological classification, for both early and advanced cancers, is the Lauren classification [27], which classifies GC according to four different types: intestinal, diffuse (signetring cell carcinoma belongs to this group), mixed, and indeterminate. The WHO classification should be also used in the pathological report. 
The EMR/ESD complete and appropriate pathological report should provide all the following items to be considered as diagnostic and clinically useful:

- Number of specimens examined (en bloc versus piecemeal resection)

- Macroscopic size of the specimen (all three dimensions should be reported)

- Macroscopic and microscopic size of the lesion

- Macroscopic tumor type

- Lauren histotype

- WHO classification with histological grade

- Depth of invasion

- Presence or absence of intratumoral ulcer

- Presence or absence of lymphovascular invasion

- Resection margins status (horizontal and vertical, with the measurement of the distance from the lesion)

- Curative resection (yes/no)

When endoscopic, macroscopic, and histological sizes of the lesion are discordant, the microscopic measure is considered the gold standard. The depth of invasion of the tumor into the submucosal layer must be measured from the deepest part of the muscularis mucosae.

The surgical pathological report of AGC should be constructed according to the following checklist:

- Type of gastrectomy and lymphadenectomy

- Tumor location

- Macroscopic type of the tumor

- Maximum tumor size

- Macroscopic distance of the lesion from the proximal and distal cut ends

- Resection margin status

- Lauren histotype

- WHO classification with histological grade

- Depth of infiltration

- Presence or absence of lymphovascular invasion

- Total number of examined lymph nodes

- Total number of positive lymph nodes

- Topography of examined and positive lymph node stations (optional)

- Peritoneal cytology or metastatic lesions (when performed)

- pTNM Classification (7th Edition)

In EGC, Kodama's classification should be also added to the pathological report.

To evaluate the histological response of the tumor to neoadjuvant therapy, the Becker classification [28] should be mentioned: grade 1, complete or subtotal regression $(<10 \%$ residual tumor per tumor bed: grade 1a is complete regression and grade $1 \mathrm{~b}$ is subtotal regression); grade 2 , partial tumor regression (10-50\% residual tumor per tumor bed); grade 3 , minimal or no tumor regression ( $>50 \%$ residual tumor per tumor bed).

\section{Adjuvant treatments and integrated therapies}

Adjuvant therapy (chemotherapy, radiotherapy, or chemoradiotherapy) could be recommended in patients surgically treated for GC at stage II-III, in R1 resection, or in case of lymph node metastases. A large meta-analysis confirmed the benefit of a 5-FU-based adjuvant treatment in stage II-III, showing a reduced 5-year mortality of $18 \%$ in the experimental group [29]. In Asian populations, an overall survival benefit from adjuvant chemotherapy was confirmed following D2 resection in the ACTS-GC trial evaluating adjuvant $\mathrm{S}-1$; the 5-year survival rate was $71.7 \%$ in the chemotherapy group versus $61.1 \%$ in the surgery-only group (HR 0.67) [30]. The CLASSIC trial evaluated an adjuvant capecitabine-oxaliplatin doublet chemotherapy after D2 gastrectomy and reported significantly improved overall survival (5-year survival rate was $78 \%$ in chemotherapy group versus $69 \%$ in the observation group) and disease-free survival (HR 0.58) with a 5 -year disease-free survival of $68 \%$ in the adjuvant chemotherapy group and $53 \%$ in the surgery-alone group [31]. However, it should be noted that the benefit of postoperative chemotherapy following a D1 or D0 lymph node dissection has not been documented in these trials.

Cytoreductive surgery (CRS) plus HIPEC represents a multidisciplinary approach for a selected subgroup of GC patients with peritoneal carcinomatosis (PC) and for advanced resectable cases at high risk of developing PC. Given that curative treatment failure in Western countries is mainly the result of peritoneal recurrence and that a metaanalysis composed almost entirely of Asian studies suggests the benefit of HIPEC as an adjuvant treatment [32], a European study on a Caucasian population is clearly warranted. In the meantime, HIPEC can be performed in selected patients having limited peritoneal carcinomatosis index $(\mathrm{PCI}<6)$ and in selected patients with metachronous PC. In cases with positive peritoneal cytology without a macroscopic peritoneal carcinomatosis and in an adjuvant setting, HIPEC would be better carried out in the context of clinical trials.

\section{Palliation}

Palliative treatment is addressed to patients affected by symptoms related to GC such as bleeding and obstruction. The main modalities of palliation are surgical procedures (resection and bypass), endoscopic therapies (stenting), bleeding control procedures (endoscopic and/or angiographic), chemotherapy, and analgesic care. The choice of modality depends on a variety of factors, including symptoms, performance status, potential response to combined therapies, and individual patient prognosis, and should be made on case-by-case basis. 
Palliative gastrojejunostomy is beneficial for gastric outlet obstruction caused by unresectable advanced distal cancer in terms of improvement of oral food intake, with acceptable morbidity and mortality. However, its indication for patients with poor performance status is less clear, and in many cases endoscopic palliation is effective as well. Reduction surgery includes gastrectomy accomplished in a metastatic disease to reduce the tumor volume and its related symptoms. This approach remains controversial. Recent results of the REGATTA trial, conducted in Asian patients, did not show any survival benefit of gastrectomy followed by chemotherapy compared with chemotherapy alone in advanced GC with a single noncurable factor, with an overall 2-year survival of $31.7 \%$ for patients treated with chemotherapy alone versus $25.1 \%$ for those treated with gastrectomy plus chemotherapy [33]. Palliative gastrectomy associated with liver resection and chemotherapy, when $\mathrm{R} 0$ resection can be obtained in patients able to endure difficult surgery, has been reported to improve overall survival in selected groups of patients [34].

In medically fit patients with metastatic or locally advanced, nonresectable, GC, chemotherapy is recommended. Chemotherapy can provide palliation, improved survival, and improved quality of life compared to best supportive care in patients with metastatic disease $[35,36]$. Currently, platinum-based and fluoropyrimidine-based combinations are accepted as first-line drug regimens [37]. Higher response rates were observed in patients who received combination chemotherapy versus monotherapy. ECF (epirubicin, cisplatin, and 5-FU) and DCF (docetaxel, cisplatin, and 5-FU) regimens are recommended as firstline chemotherapy. However, DCF was associated with increased myelosuppression and infectious complications. Oxaliplatin may represent an alternative to cisplatin with at least comparable activity and a favorable global toxicity profile. Capecitabine is an orally administered fluoropyrimidine that is converted to fluorouracil intracellularly. Several studies have evaluated capecitabine, as a single agent or in combination regimens, in patients with GC. The REAL-2 study compared capecitabine with fluorouracil and oxaliplatin with cisplatin [38]. Results from this study suggest that capecitabine and oxaliplatin are as effective as fluorouracil and cisplatin, respectively, in patients with previously untreated esophagogastric cancer with an HR 0.86 for the capecitabine-fluorouracil comparison, and an HR 0.92 for the oxaliplatin-cisplatin comparison. Irinotecan as a single agent or in combination can be an alternative when platinum-based therapy cannot be delivered.

The ToGA trial [39] showed a significant improvement in overall survival with the addition of trastuzumab to a cisplatin-fluoropyrimidine doublet. However, the benefit of trastuzumab was limited to patients with a tumor score of IHC (immunohistochemistry) $3+$ or IHC $2+$ and FISH (fluorescence in situ hybridization) positive (HR 0.74). Thus, for patients with metastatic adenocarcinoma the assessment of HER2-neu overexpression using immunohistochemistry and fluorescence in situ hybridization is recommended. The REGARD trial demonstrated a survival benefit for ramucirumab for patients with advanced gastric adenocarcinoma progressing after first-line chemotherapy (HR 0.77) [40]. Based on the results of the REGARD trial, ramucirumab as a single agent is recommended for advanced GC with disease progression, or after prior treatment by platinum-based or fluoropyrimidine-based chemotherapy.

\section{Follow-up}

There is no evidence that routine follow-up after curative treatment of GC is associated with improved long-term survival. However, routine follow-up should be offered to all patients for the following reasons: oncological (detection and management of cancer recurrence), gastroenterological (endoscopic surveillance and management of postgastrectomy symptoms), research (collection of data on treatment toxicity, time to and site of recurrence, survival, and costbenefit analyses), and pastoral (psychological and emotional support) [41, 42]. Follow-up should include lifetime monitoring of the nutritional sequelae of gastrectomy, including, but not limited to, adequate vitamin $\mathrm{B}_{12}$, iron, and calcium replacement. Follow-up should be offered by members of the multidisciplinary team who managed the initial diagnosis, staging, and treatment, including the gastroenterologist, the surgeon, the medical and radiation oncologists, and the general practitioner. Follow-up modalities should be tailored to the individual patient, to the stage of their disease, and to the treatment options available in the event that recurrence is detected. Physical examination rarely detects asymptomatic recurrence of GC; thus, a program intended to detect asymptomatic recurrence should be based on cross-sectional imaging. Upper gastrointestinal (GI) endoscopy may be used to detect local recurrence or metachronous primary GC in patients who have undergone a subtotal gastrectomy. Routine screening for asymptomatic recurrence of GC may be discontinued after 5 years, as recurrence beyond that interval is infrequent [43].

\section{EGJ}

The latest TNM classification defines junctional carcinoma as esophageal cancers, with the exception of upperthird GC not infiltrating the $\mathrm{Z}$ line. The Siewert classification, even with the limitations caused by using only a topographical definition, often not unequivocal, remains of primary importance in determining therapeutic strategies. 
In case of early junctional cancers, the en bloc endoscopic resection (EMR-ESD) should be considered therapeutic in T1a, well-differentiated, nonulcerated, and $\leq 2 \mathrm{~cm}$ lesions. In early tumors outside these criteria, endoscopic resection, even with free margins, plays only a role of staging for the high rate of lymph node metastases [44]. Thus, T1 lesions that do not meet the aforedescribed criteria should be treated with surgery; the choice of resection strategy is strictly dependent on the location with respect to the cardia: Siewert II T1 tumors can be treated with an abdominal approach if it is possible to ensure an esophageal margin of at least $2 \mathrm{~cm}$; otherwise, a thoracoabdominal approach is necessary.

In case of advanced junctional tumor, in recent years a multimodal therapy has gradually become the standard of care: for $T \geq 2$, regardless of $\mathrm{N}$, Siewert I and II, as for squamous cell carcinoma of esophagus, surgery should be preceded by neoadjuvant chemoradiotherapy [45, 46] or chemotherapy [9]. Siewert type III tumors follow the rules of advanced GC and should be treated by neoadjuvant chemotherapy. Siewert I tumors are considered tumors of the distal esophagus and the approach is the same. The best choice should be a transthoracic subtotal esophagectomy, to allow an adequate lymphadenectomy. In Siewert III tumors the procedure of choice is total gastrectomy with D2 lymphadenectomy associated with transhiatal lower mediastinal lymphadenectomy or, in selected cases, by left thoracophreno-laparotomy or right thoracotomy. A macroscopic proximal margin of at least $6 \mathrm{~cm}$ has been reported to increase the chance of surgical curability [47]; if this margin cannot be guaranteed, analysis of margin by frozen section is recommended. Siewert II tumors have the chance of having both abdominal and thoracic lymph node involvement in about one third of the cases [48]. For this reason, surgery cannot disregard a transthoracic way. The reconstruction by gastric conduit is preferable except in cases of major involvement of the stomach, where a total gastrectomy with intrathoracic esophagojejunal anastomosis should be provided.

\section{GIST}

Gastrointestinal stromal tumors (GISTs) are the most common mesenchymal tumors of the gastrointestinal tract, occurring with an incidence of at least 10-20 per million worldwide [49]. GISTs typically occur in older adults, and the median patient age ranges between 60 and 65 years. Some series have shown a mild male predominance. More than half of the GISTs occur in the stomach. Almost all GISTs express the KIT receptor tyrosine kinase, similar to the GI Cajal cells that regulate the GI autonomic nerve system and peristalsis; approximately $85-90 \%$ of GISTs contain oncogenic KIT or PDGFRA mutations. A distinct subset of GISTs, characterized by wild-type KIT/ PDGFRA, defects of succinate dehydrogenase (SDH) complex, and peculiar prognostic features, tends to occur at earlier ages, including infancy, and to prevail in females, sometimes arising in the context of Carney triad or Carney-Stratakis syndrome [50]. Most patients have symptoms or a palpable tumor at presentation, but about $25 \%$ are discovered incidentally. Tissue for pathological analysis can be obtained from tumor biopsies, done through endoscopic ultrasound guidance, or through an ultrasound/ CT-guided percutaneous approach or surgical specimens. The risk of peritoneal contamination in biopsies is minimal if the procedure is correctly carried out. Tumors at risk in this sense (e.g., cystic masses) should be biopsied only in high-volume centers. Tumor tissue should be fixed in $4 \%$ buffered formalin; Bouin's fixative should be avoided for reasons of problems of mutational analysis. The diagnosis of GIST is based on a consistent morphology associated with immunohistochemical positivity for CD117 and/or DOG1 [51]. To reduce the risk of false-positives, it is advisable to carry out the immunoreaction for CD117 without unmasking antigenic sites. About $5 \%$ of GISTs are CD117 negative. Any double negativity of CD117 and DOG1 may be a surrogate for diagnostic purposes by the finding of a 'canonical' mutation in exons 9, 11, 13, or 17 of KIT or in exons 12, 14, and 18 of PDGFRA.

Detected or suspected gastric GISTs that are $2 \mathrm{~cm}$ or more should be removed whereas smaller tumors can be excised or monitored by endoscopy and/or imaging every 6-12 months [51]. $\mathrm{R} 0$ of the tumor without rupturing the pseudo-capsule is the goal of surgery, if possible with a macroscopic margin of 1-2 cm. Gastric or esophageal GISTs should not be excised at endoscopy because R0 resection is difficult to achieve. Lymph node dissection is generally not indicated because the prevalence of lymph node metastases is about $1 \%$. Small gastric GISTs can be excised by laparoscopy by a skilled surgical team using an extraction bag.

Preoperative imatinib should be considered when an extended procedure is needed to remove the tumor. Tumor mutation analysis should be done to identify patients who do not benefit from preoperative imatinib. Five-year and 15-year recurrence-free survival rates for GISTs treated with surgery alone are estimated to be $70.5 \%$ and $59.9 \%$, respectively. Only a few tumors recurred after the first 10 years of follow-up, suggesting that most patients (about $60 \%$ ) with operable GIST are probably cured by surgery. Imatinib is the only treatment for GISTs that has been evaluated in the adjuvant setting, with results available from two randomized trials: adjuvant imatinib for at least 3 years has been recommended after surgery for high-risk patients. Patients with a small metastatic tumor burden have the longest progression-free survival times on 
imatinib treatment and, hypothetically, reduction of tumor mass by surgery might prolong the time to drug resistance. Excision of a single metastasis progressing during kinase inhibitor treatment could be considered.

The prognostic factors for GISTs are anatomic location, size, and mitotic count per $5 \mathrm{~mm}^{2}$. It should be noted that the latter value is achieved with a different number of fields at high magnification depending on the microscope used. Therefore, it is necessary to have a setup calibrated for the microscope adopted allowing for a count of $5 \mathrm{~mm}^{2}$ in place of the ambiguous 50 high-power fields (HPF) previously recommended in the literature. The combination of these parameters defines the risk of relapse. Tumor rupture in vivo (including during surgical procedures) represents another high-risk parameter, regardless of the intrinsic prognostic features of a tumor [52].

\section{Conclusions}

The guidelines reported here represent the official GIRCG position in clinical management of GC, comprehensively covering the course of the disease from diagnosis to follow up. They can be a useful tool to address physicians in managing patients with GC. According to the principles set out in these statements, physicians comply with the best, internationally accepted, actual standard of care.

\section{Compliance with ethical standards}

Conflict of interest The authors declare that they have no conflict of interest.

\section{Appendix}

\section{Recommendation for CT examination and imaging analysis}

A fasting time of at least $6 \mathrm{~h}$ is required for complete gastric emptying; $0.5-1 \mathrm{mg}$ of glucagone may be given intravenously 5-3 min before computed tomography (CT) scanning to minimize peristaltic movements (if no history of diabetes, lactose hypersensibility, or feocromocitoma (pheochromocytoma) is documented). To obtain optimal distension of the stomach we suggest oral administration of two pouches of effervescent granules (sodium bicarbonate/ citric acid) with a minimal amount of water immediately before CT scanning. Intravenous iodinated contrast agent should be injected at rate of $3-4 \mathrm{ml} / \mathrm{s}$ using a power injector, and CT images should be obtained in late arterial phase (45-50 s following the injection of contrast material), covering the entire distended stomach (from the dome of the liver to the lower limit of the distended stomach), and in portal venous phase (70-80 s following the injection of contrast material), covering the entire abdomen and thorax (from the pelvic brim to the thoracic inlet), in supine position. An MDCT unit with 16 or more rows is recommended, with the following technical parameters: effective slice thickness $1-1.5 \mathrm{~mm}$ for the arterial phase and $2.5 \mathrm{~mm}$ for the portal venous phase, beam pitch about $1 \mathrm{~mm}$, reconstruction interval about half or less than half of the effective slice thickness (to improve the quality of multiplanar 2D reconstruction); tube voltage $120-140 \mathrm{kVp}$, and reference mAs 250-500 mAs, according to the patient body mass index (BMI). Automatic tube current modulation or iterative reconstruction software should be used to minimize the radiation exposure. A standard reconstruction algorithm should be used. Compliant patients should be instructed not to breathe during helical imaging.

\section{T-staging}

To define the T-staging upon CT examination we recommended using the CT criteria proposed by Kim et al. [53]. In particular they postulated that a low-density stripe layer on a CT image indicated the submucosal layer and proper muscle layer and in turn, the inner half and the outer half of the low-density-stripe layer could be interpreted as the submucosal layer and the proper muscle layer, respectively. Taking into account this hypothesis and according to the 7th edition of the AJCC Cancer Staging Manual, we recommended the MDCT criteria reported in Table 1 for CT T-staging in GC.

Table 1 Multidetector-row computed tomography criteria for cT staging

\begin{tabular}{|c|c|}
\hline $\begin{array}{l}\text { Stage (depth of } \\
\text { invasion) }\end{array}$ & $\begin{array}{l}\text { New multidetector-row computed tomography } \\
\text { (MDCT) criteria }\end{array}$ \\
\hline T1a (mucosa) & $\begin{array}{l}\text { Tumor shows enhancement and/or thickening of } \\
\text { the inner mucosal layer, as compared to the } \\
\text { adjacent normal mucosal layer, with an intact } \\
\text { low-density stripe }\end{array}$ \\
\hline T1b (submucosa) & $\begin{array}{l}\text { Disruption of the low-density stripe layer (less } \\
\text { than } 50 \% \text { of the thickness) is visualized }\end{array}$ \\
\hline $\begin{array}{l}\mathrm{T} 2 \text { (muscularis } \\
\text { propria) }\end{array}$ & $\begin{array}{l}\text { Disruption of the low-density stripe layer } \\
\text { (greater than } 50 \% \text { of the thickness) is } \\
\text { visualized without abutting on the outer, } \\
\text { slightly high-attenuating layer }\end{array}$ \\
\hline T3 (subserosa) & $\begin{array}{l}\text { Discrimination between the enhancing gastric } \\
\text { lesion and the outer layer or a few small linear } \\
\text { strandings in the perigastric fat plane are } \\
\text { visualized }\end{array}$ \\
\hline T4a (serosa) & $\begin{array}{l}\text { An irregular or nodular outer margin of the outer } \\
\text { layer and/or a dense band-like perigastric fat } \\
\text { infiltration is visualized }\end{array}$ \\
\hline $\begin{array}{l}\text { T4b (adjacent } \\
\text { structures) }\end{array}$ & $\begin{array}{l}\text { Obliteration of the flat plane between the gastric } \\
\text { lesion and the adjacent organs or direct } \\
\text { invasion of the adjacent organs }\end{array}$ \\
\hline
\end{tabular}




\section{$N$-staging}

The results of studies evaluating the accuracy of MDCT $\mathrm{N}$-staging are somewhat disappointing. According to the meta-analysis by Kwee et al. [54], the sensitivity and specificity of MDCT N-staging varied between $62.5 \%$ and $91.9 \%$, and $50.0 \%$ and $87.9 \%$, respectively. These poor and variable results may be attributed to the lack of standard CT criteria for diagnosing metastatic lymph nodes. Although many radiologists classify malignant lymph nodes as those with short-axis diameters of $6-8 \mathrm{~mm}$ for perigastric lymph nodes, other criteria are frequently used, including roundness and central necrosis, heterogeneous or marked enhancement (more than 80 or $100 \mathrm{HU}$ ), and clustering of more than three lymph nodes. To date, the accuracy of predicting lymph node metastasis has not been satisfactory using any criteria, and there is still no consensus for diagnosing metastatic lymph nodes using CT. We recommend a double dimensional cutoff: $\geq 5 \mathrm{~mm}$ for perigastric lymph nodes (level 1 ) and $\geq 8 \mathrm{~mm}$ for the distant lymph nodes (level 2 and paraaortic), according to the distance of the lymph nodes from the primary tumor as reported in the 2nd edition of JCGC; this cutoff allows achieving an overall accuracy of $90 \%$ by the expert reader in our experience [55].

\section{References}

1. Torre LA, Bray F, Siegel RL, Ferlay J, Lortet-Tieulent J, Jemal A. Global cancer statistics, 2012. CA Cancer J Clin. 2015;65:87-108.

2. Nakajima T. Gastric cancer treatment guidelines in Japan. Gastric Cancer. 2002;5:1-5.

3. Berrino F, De Angelis R, Sant M, Rosso S, Bielska-Lasota M, Coebergh JW, et al. EUROCARE Working Group. Survival for eight major cancers and all cancers combined for European adults diagnosed in 1995-1999: results of the EUROCARE-4 study. Lancet Oncol. 2007;8:773-83.

4. De Manzoni G, Roviello F, Siquini W, editors. Surgery in the multimodal management of gastric cancer. Milan: SpringerVerlag; 2012.

5. De Manzoni G, Baiocchi GL, Framarini M, De Giuli M, D’Ugo D, Marchet A, et al. The SIC-GIRCG 2013 consensus conference on gastric cancer. Updates Surg. 2014;66:1-6.

6. Japanese Gastric Cancer Association. Japanese gastric cancer treatment guidelines 2010 (ver. 3). Gastric Cancer. 2011;14:113-23.

7. Gotoda T. Endoscopic resection of early gastric cancer. Gastric Cancer. 2007;10:1-11.

8. Montgomery M, Fukuhara S, Karpeh M, Brower S. Evidencebased review of the management of early gastric cancer. Gastroenterol Rep (Oxf). 2013;1:105-12.

9. Cunningham D, Allum WH, Stenning SP, Thompson JN, Van de Velde CJ, Nicolson M, et al. MAGIC trial participants. Perioperative chemotherapy versus surgery alone for resectable gastroesophageal cancer. N Engl J Med. 2006;355:11-20.
10. Ychou M, Boige V, Pignon JP, Conroy T, Bouché O, Lebreton G, et al. Perioperative chemotherapy compared with surgery alone for resectable gastroesophageal adenocarcinoma: an FNCLCC and FFCD multicenter phase III trial. J Clin Oncol. 2011;29:1715-21.

11. Piessen G, Messager M, Le Malicot K, Robb WB, Di Fiore F, Guilbert M, et al. Phase II/III multicentre randomised controlled trial evaluating a strategy of primary surgery and adjuvant chemotherapy versus peri-operative chemotherapy for resectable gastric signet ring cell adenocarcinomas-PRODIGE 19-FFCD1103-ADCI002. BMC Cancer. 2013;13:281.

12. Lorenzen S, Pauligk C, Homann N, Schmalenberg H, Jäger E, AlBatran SE. Feasibility of perioperative chemotherapy with infusional 5-FU, leucovorin, and oxaliplatin with (FLOT) or without (FLO) docetaxel in elderly patients with locally advanced esophagogastric cancer. Br J Cancer. 2013;108:519-26.

13. Schuhmacher C, Gretschel S, Lordick F, Reichardt P, Hohenberger W, Eisenberger $\mathrm{CF}$, et al. Neoadjuvant chemotherapy compared with surgery alone for locally advanced cancer of the stomach and cardia: European Organisation for Research and Treatment of Cancer randomized trial 40954. J Clin Oncol. 2010;28:5210-8.

14. Marrelli D, Morgagni P, de Manzoni G, Coniglio A, Marchet A, Saragoni L, et al. Italian Research Group for Gastric Cancer (IRGGC). Prognostic value of the 7th AJCC/UICC TNM classification of noncardia gastric cancer: analysis of a large series from specialized Western centers. Ann Surg. 2012;255:486-91.

15. Lee MS, Ahn SH, Lee JH, Park do J, Lee HJ, Kim HH, et al. What is the best reconstruction method after distal gastrectomy for gastric cancer? Surg Endosc. 2012;26:1539-47.

16. Hirao M, Takiguchi S, Imamura H, Yamamoto K, Kurokawa Y, Fujita J, et al. Osaka University Clinical Research Group for Gastroenterological Study. Comparison of Billroth I and Rouxen-Y reconstruction after distal gastrectomy for gastric cancer: one-year postoperative effects assessed by a multi-institutional RCT. Ann Surg Oncol. 2013;20:1591-7.

17. Sano T, Sasako M, Mizusawa J, Katayama H, Katai H, Yoshikawa $\mathrm{T}$, et al. Randomized controlled trial to evaluate splenectomy in total gastrectomy for proximal gastric carcinoma (JCOG0110): final survival analysis. J Clin Oncol 2015;33(suppl 3; abstr 103).

18. Bernini M, Bencini L, Sacchetti R, Marchet A, Cristadoro L, Pacelli F, et al. Italian Research Group for Gastric Cancer (IRGGC). The Cholegas study: safety of prophylactic cholecystectomy during gastrectomy for cancer: preliminary results of a multicentric randomized clinical trial. Gastric Cancer. 2013;16:370-6.

19. Hirao M, Kurokawa Y, Fujita J, Imamura H, Fujiwara Y, Kimura Y, et al. Osaka University Clinical Research Group for Gastroenterological Study. Long-term outcomes after prophylactic bursectomy in patients with resectable gastric cancer: final analysis of a multicenter randomized controlled trial. Surgery (St. Louis). 2015;157:1099-105.

20. Marrelli D, Pedrazzani C, Morgagni P, de Manzoni G, Pacelli F, Coniglio A, et al. Italian Research Group for Gastric Cancer. Changing clinical and pathological features of gastric cancer over time. Br J Surg. 2011;98:1273-83.

21. Roviello F, Pedrazzani C, Marrelli D, Di Leo A, Caruso S, Giacopuzzi S, et al. Super-extended (D3) lymphadenectomy in advanced gastric cancer. Eur J Surg Oncol. 2010;36:439-46.

22. Kim W, Kim HH, Han SU, Kim MC, Hyung WJ, Ryu SW, et al. Korean Laparo-endoscopic Gastrointestinal Surgery Study (KLASS) Group. Decreased morbidity of laparoscopic distal gastrectomy compared with open distal gastrectomy for stage I gastric cancer: short-term outcomes from a multicenter randomized controlled trial (KLASS-01). Ann Surg. 2016;263:28-35. 
23. Nakamura K, Katai H, Mizusawa J, Yoshikawa T, Ando M, Terashima M, et al. A phase III study of laparoscopy-assisted versus open distal gastrectomy with nodal dissection for clinical stage IA/IB gastric cancer (JCOG0912). Jpn J Clin Oncol. 2013;43:324-7.

24. Lee JH, Son SY, Lee CM, Ahn SH, Park do J, Kim HH. Morbidity and mortality after laparoscopic gastrectomy for advanced gastric cancer: results of a phase II clinical trial. Surg Endosc. 2013;27:2877-85.

25. Lauwers GY, Carneiro F, Graham DY, Curado MP, Franceschi S, Montgomery E, Tatematsu M, Hattori T. Gastric carcinoma. In: Bosman FT, Carneiro F, Hruban RH, Theise ND, editors. WHO classification of tumors of the digestive System. 4th ed. Lyon: JARC; 2010. p. 48-68.

26. Kodama Y, Inokuchi K, Soejima K, Matsusaka T, Okamura T. Growth patterns and prognosis in early gastric carcinoma. Superficially spreading and penetrating growth types. Cancer (Phila). 1983;51:320-6.

27. Lauren $P$. The two histological main types of gastric carcinoma: diffuse and so called intestinal-type carcinoma. An attempt at a histo-clinical classification. Acta Pathol Microbiol Scand. 1965;64:31-49.

28. Becker K, Langer R, Reim D, Novotny A, Meyer zum Buschenfelde C, Engel J, et al. Significance of histopathological tumor regression after neoadjuvant chemotherapy in gastric adenocarcinomas: a summary of 480 cases. Ann Surg. 2011;253:934-9.

29. GASTRIC (Global Advanced/Adjuvant Stomach Tumor Research International Collaboration) Group, Paoletti X, Oba K, Burzykowski T, Michiels S, Ohashi Y, Pignon JP, et al. Benefit of adjuvant chemotherapy for resectable gastric cancer: a metaanalysis. JAMA. 2010;303:1729-37.

30. Sasako M, Sakuramoto S, Katai H, Kinoshita T, Furukawa H, Yamaguchi T, et al. Five-year outcomes of a randomized phase III trial comparing adjuvant chemotherapy with S-1 versus surgery alone in stage II or III gastric cancer. J Clin Oncol. 2011;29:4387-93.

31. Noh SH, Park SR, Yang HK, Chung HC, Chung IJ, Kim SW, et al. CLASSIC trial investigators. Adjuvant capecitabine plus oxaliplatin for gastric cancer after D2 gastrectomy (CLASSIC): 5-year follow-up of an open-label, randomised phase 3 trial. Lancet Oncol. 2014;15:1389-96.

32. Yan TD, Black D, Sugarbaker PH, Zhu J, Yonemura Y, Petrou G, et al. A systematic review and meta-analysis of the randomized controlled trials on adjuvant intraperitoneal chemotherapy for resectable gastric cancer. Ann Surg Oncol. 2007;14:2702-13.

33. Fujitani K, Yang HK, Mizusawa J, Kim YW, Terashima M, Han SU, et al. REGATTA study investigators. Gastrectomy plus chemotherapy versus chemotherapy alone for advanced gastric cancer with a single non-curable factor (REGATTA): a phase 3, randomised controlled trial. Lancet Oncol. 2016. doi:10.1016/ S1470-2045(15)00553-7 (in press)

34. Tiberio GA, Baiocchi GL, Morgagni P, Marrelli D, Marchet A, Cipollari C, et al. Gastric cancer and synchronous hepatic metastases: is it possible to recognize candidates to R0 resection? Ann Surg Oncol. 2015;22:589-96.

35. Glimelius B, Hoffman K, Haglund U, Nyrén O, Sjödén PO. Initial or delayed chemotherapy with best supportive care in advanced gastric cancer. Ann Oncol. 1994;5:189-90.

36. Pyrhonen S, Kuitunen T, Nyandoto P, Kouri M. Randomised comparison of fluorouracil, epidoxorubicin and methotrexate (FEMTX) plus supportive care with supportive care alone in patients with non-resectable gastric cancer. $\mathrm{Br} \mathrm{J}$ Cancer. 1995;71:587-91.
37. Wagner AD, Grothe W, Haerting J, Kleber G, Grothey A, Fleig WE. Chemotherapy in advanced gastric cancer: a systematic review and meta-analysis based on aggregate data. J Clin Oncol. 2006;24:2903-9.

38. Cunningham D, Starling N, Rao S, Iveson T, Nicolson M, Coxon F, et al. Upper Gastrointestinal Clinical Studies Group of the National Cancer Research Institute of the UK. Capecitabine and oxaliplatin for advanced esophagogastric cancer. N Engl J Med. 2008;358:36-46.

39. Bang YJ, Van Cutsem E, Feyereislova A, Chung HC, Shen L, Sawaki A, et al. ToGA Trial Investigators. Trastuzumab in combination with chemotherapy versus chemotherapy alone for treatment of HER2-positive advanced gastric or gastro-oesophageal junction cancer (ToGA): a phase 3, open-label, randomised controlled trial. Lancet. 2010;376:687-97.

40. Fuchs CS, Tomasek J, Yong CJ, Dumitru F, Passalacqua R, Goswami $\mathrm{C}$, et al. REGARD Trial Investigators. Ramucirumab monotherapy for previously treated advanced gastric or gastrooesophageal junction adenocarcinoma (REGARD): an international, randomised, multicentre, placebo-controlled, phase 3 trial. Lancet. 2014;383:31-9.

41. Baiocchi GL, Marrelli D, Verlato G, Morgagni P, Giacopuzzi S, Coniglio A, et al. Follow-up after gastrectomy for cancer: an appraisal of the italian research group for gastric cancer. Ann Surg Oncol. 2014;21:2005-11.

42. Baiocchi GL, D'Ugo D, Coit D, Hardwick R, Kassab P, Nashimoto A, et al. Follow-up after gastrectomy for cancer: the Charter Scaligero Consensus Conference. Gastric Cancer. 2016;19:15-20.

43. Marrelli D, Morgagni P, de Manzoni G, Marchet A, Baiocchi GL, Giacopuzzi S, et al. Italian Research Group for Gastric Cancer. External validation of a score predictive of recurrence after radical surgery for non-cardia gastric cancer: results of a follow-up study. J Am Coll Surg. 2015;221:280-90.

44. Gronnier C, Piessen G, Mariette C. Diagnosis and treatment of non-metastatic esophagogastric junction adenocarcinoma: what are the current options? J Visc Surg. 2012;149:e23-33.

45. van Hagen $P$, Hulshof MC, van Lanschot JJ, Steyerberg EW, van Berge Henegouwen MI, Wijnhoven BP, et al. CROSS Group. Preoperative chemoradiotherapy for esophageal or junctional cancer. N Engl J Med. 2012;366:2074-84.

46. Zanoni A, Verlato G, Giacopuzzi S, Weindelmayer J, Casella F, Pasini F, et al. Neoadjuvant concurrent chemoradiotherapy for locally advanced esophageal cancer in a single high-volume center. Ann Surg Oncol. 2013;20:1993-9.

47. Ito H, Clancy TE, Osteen RT, Swanson RS, Bueno R, Sugarbaker DJ, et al. Adenocarcinoma of the gastric cardia: what is the optimal surgical approach? J Am Coll Surg. 2004;199:880-6.

48. Pedrazzani C, de Manzoni G, Marrelli D, Giacopuzzi S, Corso G, Minicozzi AM, et al. Lymph node involvement in advanced gastroesophageal junction adenocarcinoma. J Thorac Cardiovasc Surg. 2007;134:378-85.

49. Corless CL. Gastrointestinal stromal tumors: what do we know now? Mod Pathol. 2014;27(suppl 1):S1-16.

50. Miettinen M, Wang ZF, Sarlomo-Rikala M, Osuch C, Rutkowski P, Lasota J. Succinate dehydrogenase-deficient GISTs: a clinicopathologic, immunohistochemical, and molecular genetic study of 66 gastric GISTs with predilection to young age. Am J Surg Pathol. 2011;35:1712-21.

51. ESMO/European Sarcoma Network Working Group. Gastrointestinal stromal tumors: ESMO Clinical Practice Guidelines for diagnosis, treatment and follow-up. Ann Oncol. 2014;23(suppl 3):iii21-6. 
52. Joensuu H. Risk stratification of patients diagnosed with gastrointestinal stromal tumor. Hum Pathol. 2008;39:1411-9.

53. Kim JW, Shin SS, Heo SH, Choi YD, Lim HS, Park YK, et al. Diagnostic performance of 64-section CT using CT gastrography in preoperative T-staging of gastric cancer according to 7th edition of AJCC cancer staging manual. Eur Radiol. 2012;22:654-62.
54. Kwee RM, Kwee TC. Imaging in assessing lymph node status in gastric cancer. Gastric Cancer. 2009;12:6-22.

55. Marrelli D, Mazzei MA, Pedrazzani C, Di Martino M, Vindigni C, Corso G, et al. High accuracy of multislices computed tomography (MSCT) for para-aortic lymph node metastases from gastric cancer: a prospective single-center study. Ann Surg Oncol. 2011;18:2265-72. 